\title{
Denoising data acquisition algorithm for array pixelated CdZnTe nuclear detector
}

https://doi.org/10.1515/phys-2019-0015

Received Oct 29, 2018; accepted Jan 28, 2019

\begin{abstract}
Traditionally, the binary search method is used to collect the denoising data in the array pixilated CdZnTe nuclear detector. Due to the high dispersion of the data itself, the acquisition efficiency is low and the acquisition result has a large error. A denoising data acquisition algorithm for array pixilated CdZnTe nuclear detector is proposed. The detector principle and system noise type are analyzed. The buffer half-full storage algorithm and multithread control method are used to collect the noise data of array pixilated CdZnTe nuclear detector. The experimental data show that the proposed algorithm can effectively collect the denoising data of the array pixilated CdZnTe nuclear detector, and the acquisition error rate is only 0.25 , the acquisition speed growth rate is up to $96 \%$, with high acquisition accuracy and efficiency.
\end{abstract}

Keywords: CdZnTe, array pixelated nuclear detector, denoising data, acquisition algorithm, buffer half-full storage algorithm, multi-thread control

PACS: 29.40.-n, 29.85.Fj, 29.85.Ca

\section{Introduction}

High-energy ray energy spectrum detection and imaging detection technology are the key technology in the fields of atomic energy science, space science, materials science and biomedical science. It has important applications in nuclear energy utilization, radiation protection, nuclear safety testing, etc. [1]. Since the signing of the

\footnotetext{
`Corresponding Author: Xiangxiang Luo: Northwestern Polytechnical University, School of Materials Science and Engineering, Xi’an, 710072 China; Email: tjjlwh668866@163.com

Ruiqi Hu: Northwestern Polytechnical University, School of Materials Science and Engineering, Xi'an, 710072 China;

Email: luo_xiangx@163.com

Nasruddin Hassan: School of Mathematical Sciences, Faculty of Science and Technology, University Kebangsaan Malaysia UKM Bangi, Selangor, Malaysia; Email: nas@ukm.edu.my
}

¿ Open Access. (c) 2019 X. Luo et al., published by De Gruyter. (Cc) BY License
CTBTO, passive and portable nuclear safety testing in current international cooperation and exchange is an important research area of national defense security, and highperformance radiation detection technology is urgently needed. CdZnTe, a new material for room temperature semiconductor radiation detectors with excellent performance, has attracted widespread attention in the field of nuclear radiation detection at home and abroad [2]. The CdZnTe radiation detector made of CdZnTe material is a new generation of nuclear radiation detector after Nal, Csl and HPGe detectors. It has broad prospects and plays an important role in the research fields of high energy nuclear physics, heavy ion physics, atomic physics, astrophysics and so on [3]. Compared with the conventional planar electrode CdZnTe detector, the CdZnTe detector with unipolar sensitivity has a higher detection efficiency and energy resolution. The surface pixelated array CdZnTe crystal can be prepared into a nuclear radiation imaging detector with multi-purpose, wide-spectrum, passive three-dimensional position sensitivity. It has important applications in nuclear radiation detection and nuclear safety as well as nondestructive testing and nuclear medicine imaging. Many researchers have studied the meta-pixel array CdZnTe detector [4]. Wojenski et al. [5] proposed a signal acquisition method of SXR spectroscopy system gemstone detector based on FPGA, but it was only suitable for neutron radiation, strong electromagnetic fields and other environments, with certain limitations. Tuoriniemi et al. [6] proposed a new algorithm for peak identification, which was used to quickly resolve single particles in data. It was difficult to collect ultra-fine nanoparticles during acquisition, resulting in low acquisition accuracy. Cheong et al. [7] used the binary search method for data acquisition. The acquisition efficiency was low due to the high dispersion of the data itself. In this paper, the denoising data acquisition algorithm in array pixelated CdZnTe nuclear radiation detection system is studied. Through data verification, the algorithm has a high application value.

This work is licensed under the Creative Commons Attribution 4.0 


\section{Researchon denoising data acquisition algorithm for array pixelated CdZnTe nuclear detector}

\subsection{Detector principle and system noise identification}

The array pixelated CdZnTe detector is designed by a single-sided block electrode of CdZnTe crystal [8] to form anarray pixelated electrode structure on the surface of the crystal anode, and the cathode is an integral planar electrode. When the detector is working, a negative high voltage is applied to the surface of the cathode. Under the electric field formed by the applied bias voltage, a large number of electrons and holes generated by the interaction of the radiation and the crystal migrate to the two poles, respectively [9]. The induced charge generated by the pixel is collected by the pixel electrode and converted into a voltage pulse signal whose amplitude is proportional to the in cident photon energy, and then further shaped and amplified by the shaping amplifier to obtain a pulse signal with a higher signal to noise ratio. The corresponding energy spectrum distribution is obtained by statistic of the signal pulse amplitude distribution [10].

For an ideal semiconductor nuclear radiation detector system, all pulse signals of a single energy ray correspond to the same peak signal path. However, in actual detection, there must be a certain detection system noise, which leads to the broadening of the spectrum of the detection system [11]. Therefore, reducing the various noise effects of the detection system has always been the focus of research in the field of semiconductor nuclear radiation detectors [12].

The total equivalent noise $(\mathrm{eV})$ of a semiconductor detector system can be expressed as:

$$
\Gamma_{\text {total }}^{2}=\Gamma_{\mathrm{F}}^{2}+\Gamma_{\mathrm{col}}^{2}+\Gamma_{\mathrm{e}}^{2}
$$

$\Gamma_{\mathrm{F}}$ is the fluctuation noise of the number of electron-hole pairs generated in the crystal, which is related to the nature of the detector material. The specific values are:

$$
\Gamma_{\mathrm{F}}=2.355 \overline{F w E_{\text {in }}}
$$

where $F$ is the Fano factor, $w$ is the average ionization energy of the CdZnTe crystal, and $E_{\text {in }}$ is the ray energy. The fluctuation noise of charge number $\Gamma_{\mathrm{F}}$ determines the limit of the energy resolution that the detector can achieve, that is, the inherent limit energy resolution. For a detector using CdZnTe crystal as a material, the Fanofactor $F=0.14$, the average ionization energy $w=4.64$, and when the incident photon energy is $60 \mathrm{keV}$, the fluctuation noise of charge number $\Gamma_{\mathrm{F}}=0.464 \mathrm{keV}$. Therefore, the CdZnTe detector has a small fluctuation noise of charge number, and its inherent limit energy resolution is high.

$\Gamma_{\text {col }}$ is the incomplete noise of charge collection, indicating that electrons and holes are recombined or captured during the migration process, so that the carrier charge mobile phone is incomplete and generates noise. For the ideal conditions of high energy radiation conditions $(\mathrm{MeV})$ and charge cell phone efficiency $\eta>0.9$, the semi-empirical equation of $\Gamma_{\mathrm{col}}$ is:

$$
\Gamma_{\text {col }}=4.7 \times 10^{5}(1-\eta)\left(E_{\text {in }}\right)^{\frac{1}{2}}
$$

where $E_{\text {in }}$ is the ray energy and $\eta$ is the charge collection efficiency. Although the array pixelated CdZnTe detector has a "small pixel effect" in the inductive charge collection process, the small array pixelated electrode structure of the anode makes the induced charge collected by the detector mainly depend on the electron migration motion near the small pixel anode [13], while the contribution of hole migration inside the crystal to the output signal is significantly reduced, the effect of hole trapping on the detector's induced charge signal collection is also weakened, and the "low energy tail" is improved [14], but in fact, considering the diffusion effect of carriers, the incomplete noise $\Gamma_{\text {col }}$ of charge collection is still the main source of noise for array pixelated CdZnTe detector systems.

$\Gamma_{\mathrm{e}}$ is the equivalent electronic noise of the detection system. It is the main source of noise in the detector system and can be expressed as:

$$
\Gamma_{\mathrm{e}}^{2}=\Gamma_{\text {pre }}^{2}+1.18 \times 10^{3} I_{d} \tau+0.10 \frac{T}{\tau} r_{s}\left(C_{d}^{2}+2 C_{d} C_{0}\right)
$$

where $\Gamma_{\text {pre }}$ is the equivalent noise of the charge sensitive preamplifier itself, $I_{d}$ is the leakage current of the CdZnTe crystal, $T$ is the ambient temperature, $\tau$ is the time constant of signal forming, and $r_{s}$ is the equivalent input impedance of the preamplifier FET, $C_{d}$ is the source-gate capacitance $(\mathrm{pF})$ of the preamplifier. It can be seen from the above equation that the equivalent electronic noise of the readout circuit and the leakage current noise of the crystal surface electrode are the main influencing factors of the electronic noise of the detection system [15].

The noise of the array pixelated CdZnTe detection system mainly comes from the incomplete noise of charge collection, the equivalent electronic noise of the readout circuit, and the leakage current noise of the crystal surface electrode. Among them, the incomplete noise of charge collection will cause a severe low-energy tail effect in the measured energy spectrum, and the equivalent electronic 
noise of the readout circuit and the leakage current noise of the crystal surface electrode will broaden the symmetry broadening peak and reduce the energy resolution rate of the detection system [16]. There are different noise sources in most electronic components: thermal noise, low frequency voltage noise, shot noise, and noise in transistors. Noise can be thought of as a macroscopic representation of a microscopic stochastic process, so it can be derived by considering microscopic processes [17]. The amplitude of the equivalent noise of the preamplifier is affected by many factors, and its interference noise is inherently inevitable [18]. The main work of this paper is aimed at collecting system noise [19].

\subsubsection{Thermal noise}

Due to the thermal fluctuation of the electron distribution in the conductor, we can consider a resistor with a resistance of $R$. When no current flows, the noise voltage $U_{n}$ can still be measured across the resistor. Calculating the noise power spectral density based on thermodynamics can be given by:

$$
\frac{\overline{d U_{n}^{2}}}{d f}=4 K T R
$$

where $f$ is the frequency; $K$ is the Boltzmann constant; $T$ is the absolute temperature.

According to equation (5), a physical resistor can be described as an ideal noise-free resistor $R$ connected in series with the same noise voltage source or in parallel with the same noise current source. The specific spectral density is given by:

$$
\begin{aligned}
& \frac{\overline{d U_{n}^{2}}}{d f}=4 K T R \\
& \frac{\overline{d I_{n}^{2}}}{d f}=\frac{4 K T}{R}
\end{aligned}
$$

\subsubsection{Low frequency voltage noise}

This type of noise can be seen in most electronic devices. In most cases, the noise power spectrum has an approximate 1/f relationship, which can be expressed by:

$$
\frac{d U_{n}^{2}}{d f}=\frac{A_{n}}{f}
$$

The physical source of this noise is not unique and has many different mechanisms for different types of electronic components. And for the same electronic components, the noise intensity is related to the details of the technical production process [20].

\subsubsection{Shot noise}

Shot noise is a result of the electron point and discontinuity properties and represents a fluctuation of the number of carriers that make up the charge $Q=N q$. Considering a "constant" current of $I$, we expect the charge $\Delta Q=I \Delta t$, which flows through the boundary along the current path within a (short) time interval $\Delta t$. This is equivalent to an average of $\Delta N=I \Delta t / q$ electrons with a fluctuation of $\delta \Delta N=\sqrt{\Delta N}$, so the mean square change of the measured current in a short time interval is:

$$
\left\langle\delta I^{2}\right\rangle=\frac{q^{2}(\delta \Delta N)^{2}}{\Delta t^{2}}=\frac{q^{2} \Delta N}{\Delta t^{2}}=\frac{q I}{\Delta t}
$$

From these relationships, the frequency spectrum of the noise current is derived to obtain the following equation:

$$
\frac{d\left\langle i_{n}^{2}\right\rangle}{d f}=2 I q
$$

The derivation of this equation makes the implicit assumption that the probability of an electron passing through a boundary is independent of other electrons. This illustrates the change in potential distribution caused by electrons that have crossed the boundary [21], without affecting the probability of other electrons crossing the boundary. Generally, this condition can be satisfied. In addition, shot noise requires current generated by an external power source, while thermal noise can exist in the device even without an external power supply [22].

\subsubsection{Noise in the transistor}

Thermal noise is important in all types of transistors. Shot noise is important in bipolar transistors because their base current is only two orders of magnitude lower than the pole current, the fluctuation of the base current is negligible compared to the signal current. In junction field effect transistors, shot noise dominates. In $1 / f$ Noise, MOSFET is very significant, but not so important in JFETs and dual transistors.

The thermal noise in the field effect transistor is the thermal noise generated by the resistance of the channel. The thermal noise here cannot be simply calculated by the above equation, and more complicated analysis is needed to consider the interaction of the channel with the gate. The physical cause of low frequency noise in unipolar transistors is crystal defects, which cause carrier trapping. The carrier local area of the motion is captured and is not released after a certain delay. Because it is captured, the presence of all transiently fixed charges will sense the channel charge and thus modulate the crystal current. 


\subsection{Denoising data acquisition algorithm for array pixelated CdZnTe nuclear detector}

Based on the analysis of the principle of the array pixelated CdZnTe nuclear detector and the identification of the noise inside the detector, the buffer half-full storage algorithmis used for the denoising data acquisition in the detector. Compared with the buffer algorithm and data compression algorithm in the existing data acquisition system, the biggest advantage of this algorithm is that the denoised data collected is not easily lost, and the integrity of the denoised data can be guaranteed. At the same time, the real-time multi-task control method is used in the detector, and the collected data is saved directly into the file in binary form. This not only achieves the purpose of large storage capacity and high data transmission efficiency, but also satisfies the requirements for real-time image transmission [23].

The buffer half-full storage algorithm is used to set a data HFIFO buffer pointer in the data acquisition process. The buffer size is set to 8192 bytes. When data appears on the PC104 bus, the received data frame is first placed in the HFIFO buffer. When the HFIFO reaches half-full, the lower 4096 bytes of data are read. While reading out the data, it makes room for the HFIFO, and the data frame received in the bus board continues to be placed in the HFIFO buffer. The specific operations are as follows:

Firstly, the buffer is divided into the following five states:

State 0 indicates that the HFIFO is empty.

State 1 indicates that the HFIFO is not empty but less than half-full

State 2 indicates that the HFIFO has reached or exceeded half-full but has not reached full

State 3 indicates that the HFIFO is full

State 4 indicates an unknown state and should not appear

Secondly, the state of the HFIFO is judged:

(a) When the data does not reach half of the buffer size (i.e., state 0 or state 1): return at this time to perform denoising data acquisition.

(b) When the data reaches or exceeds half of the buffer size but is not full (i.e., state $=2$ ): the process will first judge the HFIFO state, and if it is half-full, read all 4096 bytes of data at a time; If it is more than halffull, but not full, at this time, only the lower 4096 bytes of data are read, and the original high data is advanced, occupying the HFIFO low position. All the data read out is saved directly to the binary file.
In particular, it is proposed that since the multisensor detection system has a long acquisition time or no time limit [24], the collected data is saved directly into the file in the acquisition algorithm design, which saves a lot of time in storage engineering. In addition, the data is stored in a binary file, enabling real-time display of images.

(c) When the HFIFO is full (i.e., state 3): At this point, the data acquisition process can return as long as the HFIFO is read empty or the number specified by the user is reached. When the reading is divided into two batches, the lower 4096 bytes of data are first read and saved into the binary file; then the upper data is read out, that is, the HFIFO is read, and the previous data is written into the file.

The advantages of the two batches of readings are as follows: (1) shortening the time of one reading; (2) after reading the low byte data, make room for the HFIFO so that the data frames received in the bus board continue to put into the HFIFO buffer, the data is not easy to lose, ensuring the integrity of the data.

(d) Other values: indicates an unknown state and should not appear. It will not be described in detail here.

Multi-thread control: Since the detector is composed of multiple detection modules, the denoising data acquisition algorithm detects and controls the multi-path parameters in the detector. In order to reduce the complexity of the whole system, realize real-time image transmission, meet the high-speed requirements and interrupt requirements of the acquisition process, and ensure that the frame rate reaches or exceeds $100 \mathrm{~Hz}$, the data acquisition algorithm needs multi-task parallelism, that is, design multiple threads. And because of the different tasks, the design of each thread is not the same [25-31]. The algorithm has a total design including data acquisition thread, control thread, and drawing thread. When the program runs at most, several threads may run in parallel. To ensure the integrity of a large amount of data and error correction recovery, the data collection thread has a higher priority than the control thread. The structure diagram is shown in Figure 1.

As shown in Figure 1, the HFIFO buffer enters drawing thread 2 in state 2.

Drawing thread 1: When drawing, it needs to judge the HFIFO status first. If it is half-full, read the first 4 frames, that is, the lower 4096 pixels, then return immediately to draw. Only the first is frame drawn when drawing, and finally the data is saved. The HFIFO buffer enters drawing thread 2 in state 3. Drawing thread 2: 8 frames are read at 


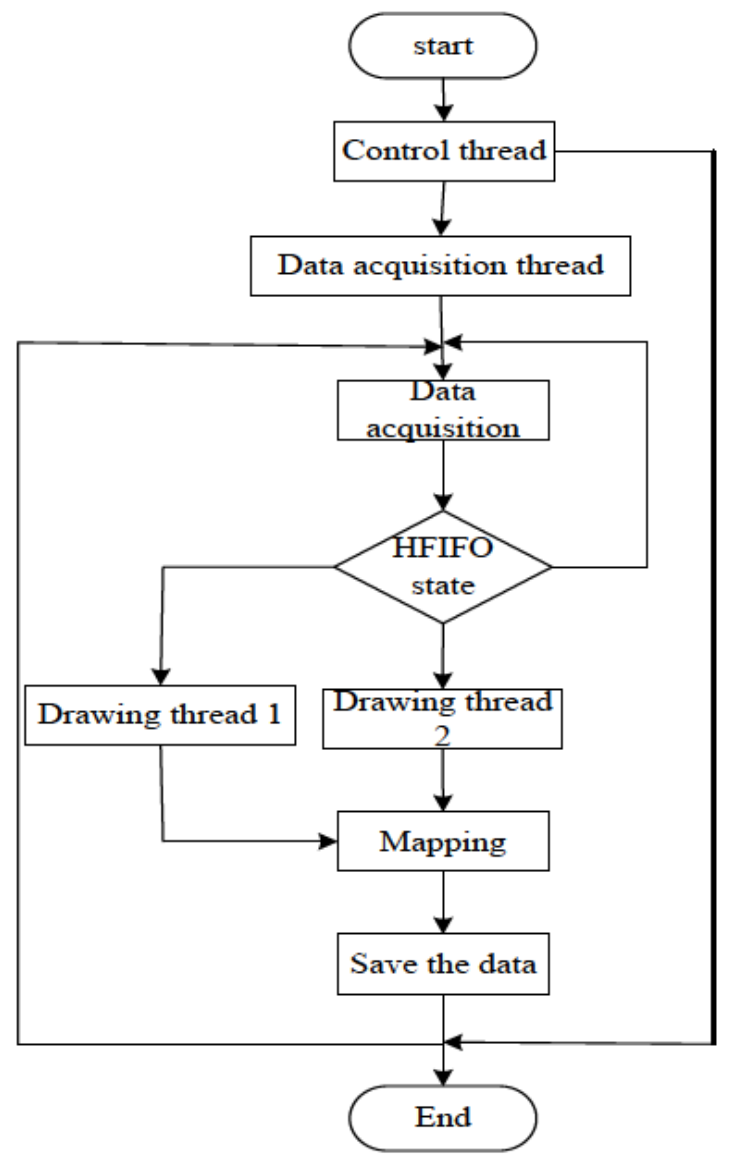

Figure 1: Algorithm structure diagram

a time, that is, 8192 pixels, and then immediately return to draw. Only the first is frame drawn when drawing, and finally the data is saved, the drawing thread is shown in Figure 2:

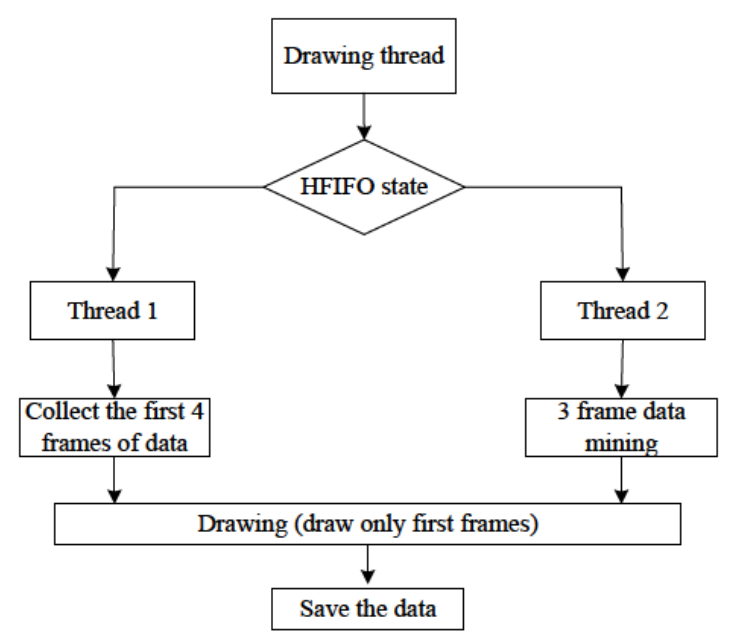

Figure 2: Drawing thread diagram

\section{Results}

\subsection{Analysis results of algorithm's validity}

Due to the "edge effect" of the edge pixels of the array pixelated CdZnTe detector, the performance of the detector's edge pixels determines the effective range of the array pixelated imaging detector. Therefore, if the detection system can improve the response signal of the pixel at the edge of the crystal, a large-area array pixelated CdZnTe nuclear imaging system with excellent performance can be established by repeatedly preparing the signal processing channel. Based on the above considerations, the system performance test is carried out on the $2 \times 2$ pixels of the crystal corner under the algorithm using the $59.5 \mathrm{keVAm}$ gamma source. The bias voltage is $-600 \mathrm{~V}$ and the detection time is 10 minutes. The energy spectrum of the pixel at the edge of the crystal is obtained. Figure 3 shows the peak results of the corresponding pulses output by the experimental detection system.

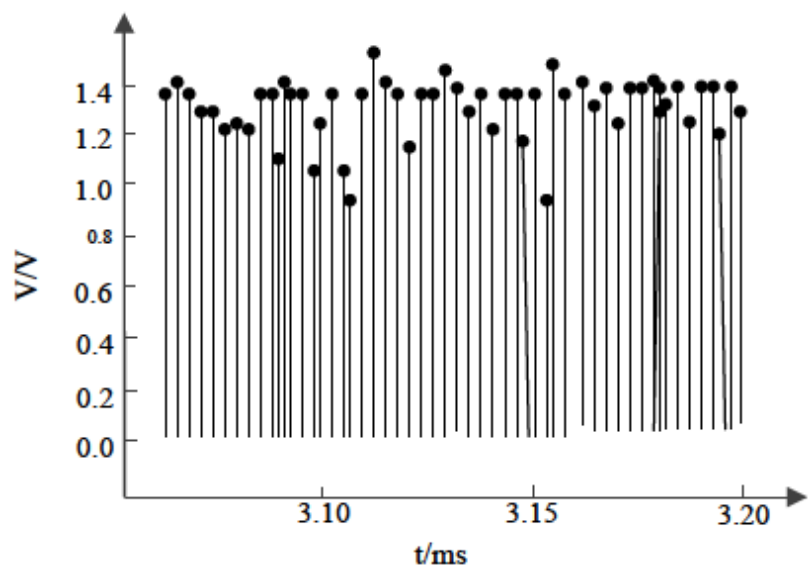

Figure 3: Peak finding of the pulse signal

As shown in Figure 3, the noise of the output pulse signal of the experimental detection system is small, the signal is not stacked and the peak statistics are accurate. The electronic noise of the readout circuit of the detection system is well suppressed.

Figure 4 shows the edge pixel energy spectrum with the highest energy resolution of the experimental detection system under the proposed algorithm.

As shown in Figure 4, due to the short detection time and the influence of crystal edge effect, the event counts of low energy and omnipotent peaks in the energy spectrum distribution are small, and the escape peak of 36.1 $\mathrm{keV} \mathrm{Cd}$ is not obvious, and the mixed peak $K_{\alpha 1, \alpha 2}$ of Teat 


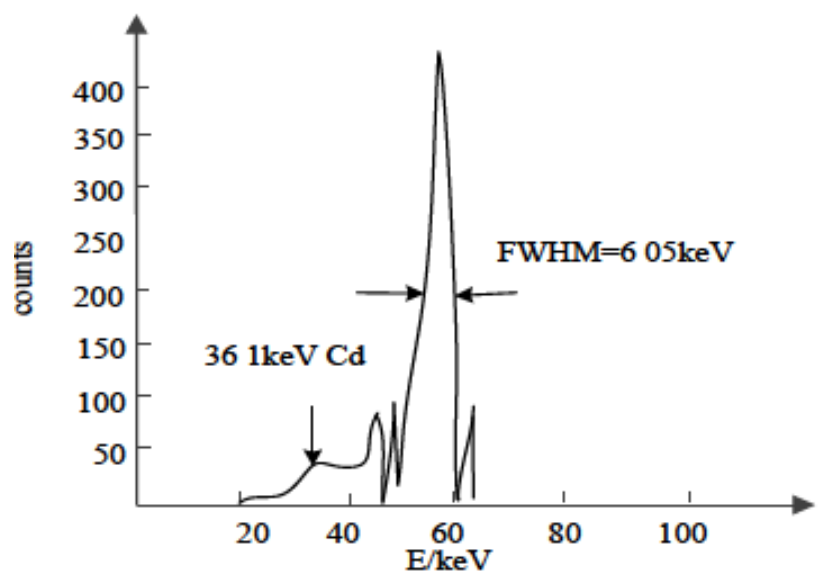

Figure 4: Energy spectrum of $241 \mathrm{Am}(59.5 \mathrm{keV})$

$27.4 \mathrm{keV}$ cannot be confirmed because of the higher threshold setting during pulse peaking. The obtained pluripotent peak is approximately symmetric and the low energy tail is not obvious. It indicates that the incomplete noise of charge collection and the surface leakage current noise of the CdZnTe crystal in the detection system using the proposed algorithm are improved, and the obtained peak spectrum $F W H M=6.05 \mathrm{keV}$. The energy resolution is $10.08 \%$, and the energy resolution performance of the entire pixel array detector has been significantly improved. The detection performance of the edge pixel signal of the array pixelated CdZnTe crystal meets the requirements of nuclear radiation detection.

A comprehensive analysis of the above results shows that the proposed algorithm can effectively collect the noise data in the array pixelated CdZnTedetector, so as to obtain the peak finding result and the edge pixel energy spectrum with the highest energy resolution.

In order to further verify the performance of the buffer half-full storage algorithm used to collect the denoised data in the array pixelated CdZnTe detector, the peak identification algorithm, the binary search algorithm and the proposed algorithm are compared.

\subsection{Analysis results of algorithm's performance}

\subsubsection{Analysis results of algorithm's robustness}

The proposed algorithm, peak identification algorithm and binary search algorithm are tested to collect the denoising data in the array pixelated CdZnTe detector, and the robustness of the three algorithms is compared. The number of experiments is 7 and the results are depicted in Figure 5:

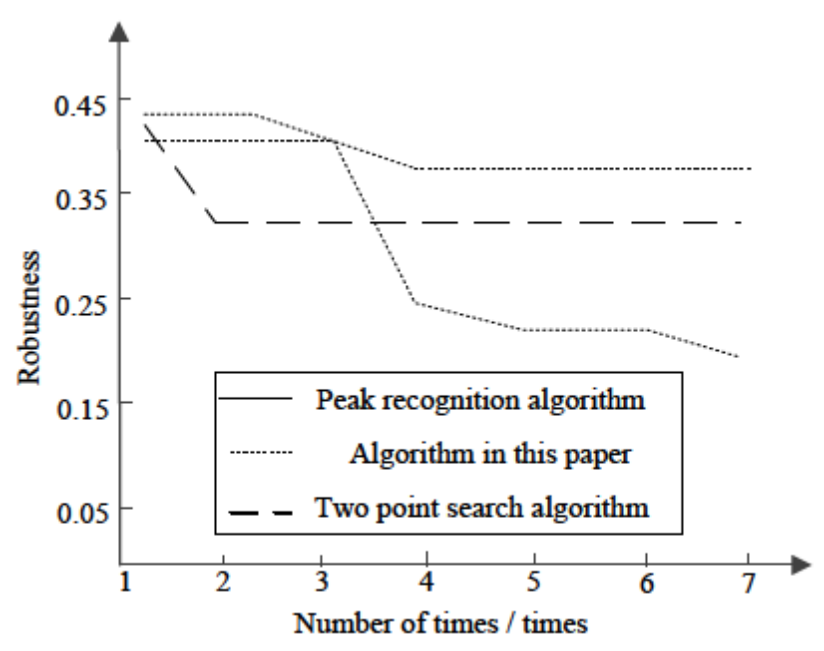

Figure 5: Robustness test results

Analysis of Figure 5 shows that the number of experiments is 7 times. With the increase of the number of experiments, the trend of the proposed algorithm has slight fluctuations. It is always above the peak identification algorithm and the binary search algorithm. In the third experiment, it has coincidence with the peak identification algorithm, and the data shows that the robustness of the proposed algorithm is 0.44 and the minimum is 0.40 . The maximum robustness of the peak identification algorithm is 0.43 and the minimum is 0.17 . The maximum robustness of the binary search algorithm is 0.44 and the minimum is 0.33. It can be seen from the data comparison that the robustness of the proposed algorithm is the best. It describes whether the algorithm process and the result are stable when the algorithm is in an abnormal and dangerous situation. The proposed algorithm has good stability when collecting denoising data.

\subsubsection{Error rate analysis results of the algorithm for denoising data acquisition}

Figure 6 shows the denoising data errors in the three algorithms for the acquisition of array pixelated CdZnTe detector with different amounts of denoised data. The minimum number and the maximum number of denoised data are 10 and 40, respectively.

Analysis of Figure 6 shows that there are 40 denoising data in the array pixelated CdZnTe detector. With the increase of the denoising number, the acquisition error of 


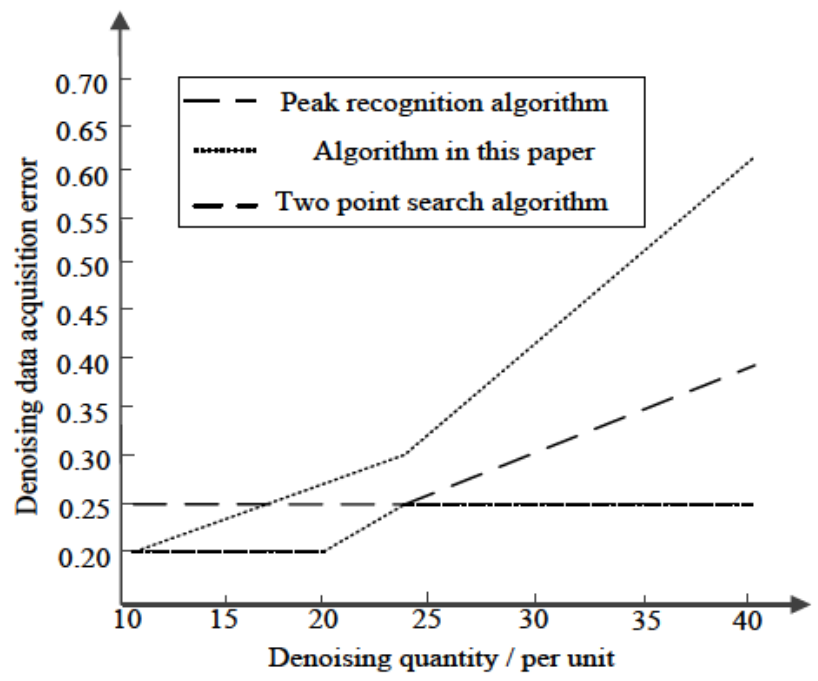

Figure 6: Denoising data acquisition error test results

the proposed algorithm is less fluctuating. According to the data, the maximum error rate of the denoising data acquisition in the array pixelated CdZnTe detector by using the proposed algorithm is 0.25 , the minimum value is 0.20 ; while that of the peak identification algorithm is 0.65 , the minimum value is 0.20 ; and the maximum value of the error rate by the binary search method is 0.40 and the minimum value is 0.25 . It can be seen from the data comparison that the proposed algorithm has the minimum error in the acquisition of denoising data in array pixelated CdZnTe detector. The algorithm has a high accuracy.

\subsubsection{Analysis results of the acquisition efficiency of the algorithm}

The comparison results of the denoising data acquisition efficiency of the three algorithms in the array pixelated CdZnTe detector under different experimental times are described in Figure 7.

Analysis of Figure 7 shows that in the comparison of the acquisition speed's growth rate of the three algorithms, the overall trend of the acquisition speed's growth rate of the proposed algorithm is above the other two algorithms, the maximum growth rate of the proposed algorithm is $96 \%$, while the maximum growth rate of peak identification algorithm is $95 \%$, and the maximum growth rate of the acquisition speed of the binary search method is $91.8 \%$. It can be seen from the data comparison that the proposed algorithm has a faster growth rate and higher efficiency for the denoising data acquisition in the array pixelated CdZnTe detector.

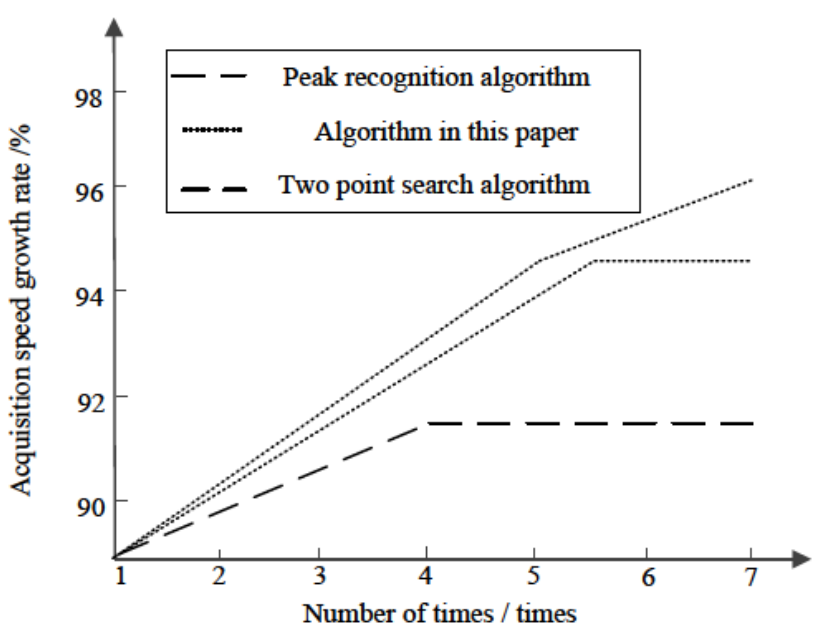

Figure 7: Algorithm acquisition speed growth rate test results

The time-consuming situation of the denoising data acquisition of the three algorithms in the array pixelated CdZnTe detectoris count. Eight groups of experiments are set up, and the number of denoising in each group is different, and four data acquisitions are performed. The results are shown in Tables 1, 2 and 3.

Table 1: Time-consuming test results of peak recognition algorithm for noise removal data acquisition

\begin{tabular}{cccccc}
\hline Denoising & \multicolumn{5}{c}{ Acquisition time consuming /s } \\
\cline { 2 - 6 } $\begin{array}{c}\text { quantity / } \\
\text { per unit }\end{array}$ & $\begin{array}{c}\text { First } \\
\text { times }\end{array}$ & $\begin{array}{c}\text { Second } \\
\text { times }\end{array}$ & $\begin{array}{c}\text { Third } \\
\text { times }\end{array}$ & $\begin{array}{c}\text { Fourth } \\
\text { times }\end{array}$ & $\begin{array}{c}\text { Time per } \\
\text { request }\end{array}$ \\
\hline 100 & 168 & 168 & 167.5 & 167.4 & 167.73 \\
200 & 336 & 336 & 335 & 334.8 & 335.45 \\
300 & 504 & 504 & 502.5 & 502.2 & 503.18 \\
400 & 672 & 672 & 670 & 669.6 & 670.9 \\
500 & 840 & 840 & 837.5 & 837 & 838.63 \\
600 & 1008 & 1008 & 1005 & 1004.4 & 1006.35 \\
700 & 1176 & 1176 & 1172.5 & 1171.8 & 1174.08 \\
800 & 1344 & 1344 & 1340 & 1339.2 & 1341.8 \\
Mean value & 756 & 756 & 753.75 & 753.3 & 754.765 \\
\hline
\end{tabular}

It can be seen from Tables 1,2 and 3 that in the timeconsuming comparison results of the denoising data acquisition by using the three algorithms, eight sets of denoising data are collected four times, and a total of 800 denoising data are collected. It can be seen that the average acquisition time of each denoising data by using the peak recognition algorithm is about 753s, and the average time of the binary search method for 800 denoising data is about 649s. The average acquisition time of the proposed algorithm for different denoising data is about 540s. The analysis can be concluded that compared with the other 
Table 2: The time-consuming test results of the algorithm in data collection

\begin{tabular}{cccccc}
\hline Denoising & \multicolumn{5}{c}{ Acquisition time consuming /s } \\
\cline { 2 - 6 } $\begin{array}{c}\text { quantity / } \\
\text { per unit }\end{array}$ & $\begin{array}{c}\text { First } \\
\text { times }\end{array}$ & $\begin{array}{c}\text { Second } \\
\text { times }\end{array}$ & $\begin{array}{c}\text { Third } \\
\text { times }\end{array}$ & $\begin{array}{c}\text { Fourth } \\
\text { times }\end{array}$ & $\begin{array}{c}\text { Time per } \\
\text { request }\end{array}$ \\
\hline 100 & 120 & 120 & 120 & 120.5 & 120.13 \\
200 & 240 & 240 & 240 & 241 & 240.25 \\
300 & 360 & 360 & 360 & 361.5 & 360.13 \\
400 & 480 & 480 & 480 & 482 & 480.5 \\
500 & 600 & 600 & 600 & 602.5 & 600.63 \\
600 & 720 & 720 & 720 & 723 & 720.75 \\
700 & 840 & 840 & 840 & 843.5 & 840.88 \\
800 & 960 & 960 & 960 & 964 & 961 \\
Mean value & 540 & 540 & 540 & 542.25 & 540.54 \\
\hline
\end{tabular}

Table 3: The time-consuming test results of the two point search method for noise elimination data acquisition

\begin{tabular}{cccccc}
\hline Denoising & \multicolumn{5}{c}{ Acquisition time consuming /s } \\
\cline { 2 - 6 } quantity / & First & Second & Third & Fourth & Time per \\
per unit & times & times & times & times & request \\
\hline 100 & 144 & 144.5 & 144.5 & 1144.5 & 144.38 \\
200 & 288 & 289 & 289 & 289 & 288.75 \\
300 & 432 & 433.5 & 433.5 & 433.5 & 433.13 \\
400 & 576 & 578 & 578 & 578 & 577.5 \\
500 & 720 & 722.5 & 722.5 & 722.5 & 721.88 \\
600 & 864 & 867 & 867 & 867 & 866.25 \\
700 & 1008 & 1011.5 & 1011.5 & 1011.5 & 1010.63 \\
800 & 1152 & 1156 & 1156 & 1156 & 1155 \\
Mean value & 648 & 650.25 & 650.25 & 775.25 & 649.69 \\
\hline
\end{tabular}

two algorithms, the propsoed algorithm has the lowest average acquisition time for different denoising data. The algorithm has the advantage of high acquisition efficiency.

In summary, when the denoising data is collected in the array pixelated CdZnTe detector, the stability of the proposed algorithm is higher, the denoising data acquisition error is aminimum, and the denoising data acquisition efficiency is the highest.

\section{Discussion}

In view of the research content in this paper, the following suggestions are proposed for data collection in future detectors:

Improvement of CdZnTe crystal electrode structure. According to the research on CdZnTe detectors at home and abroad, the improvement of the electrode structure of CdZnTe crystal has the most direct impact on the performance of the detector. Therefore, it is possible to study and design a more efficient pixel array microelectrode to change the internal weight distribution of the crystal, thereby improving the carrier collection capability of the anode of the array pixelated detector; or on the basis of the existing surface pixel electrode structure, different forms of protective gates are studied and prepared to improve the defects of large pixel leakage current at the edge of the CdZnTe crystal.

Detector Signal Processing.

It is possible to further study the whole set of signal processing algorithms with pulse peak finding, rise time discrimination and peak correction of energy spectrum characteristics, which will greatly improve the detection efficiency of the detection system.

Study on Electric Field Distribution in CdZnTe crystal. Based on the work of this paper, the CdZnTe detector induced signal model considering the carrier diffusion effect can be further established to expand the application range of the CdZnTe detector's imaging evaluation model. Aiming at the internal electric field distribution of CdZnTe crystal under low energy and low voltage conditions, a multiphysics coupled electric field distribution model combined with weight potential theory is established and studied. In the experimental aspect, the internal electric field distribution image of the crystal during the polarization effect can be further observed by the Pockels effect to establish a more perfect electric field distribution variation theory in the CdZnTe crystal.

\section{Conclusions}

In this paper, the denoising data acquisition algorithm of array pixelated CdZnTe nuclear detector is proposed. Firstly, the detector principle and system noise are analyzed. Secondly, the buffer half-full storage algorithm is used to collect the denoising data in the detector. Experimental data verification shows that the proposed algorithm can effectively collect the denoising data of the array pixelated CdZnTe nuclear detector, the acquisition error rate is only 0.25 , and the acquisition speed growth rate is as high as $96 \%$. When the proposed algorithm performs denoising data acquisition, it has high stability and high efficiency, and the error of denoising data acquisition is small, which has high use value.

Acknowledgement: This work was supported by the Special Fund of National Key Scientific Instruments and Equipments Development (2011YQ040082), the National 973 Project of China (2011CB610400), the 111 Project of China (B08040), the National Key Research and Develop- 
ment Program of China (2016YFF0101301), the National Natural Science Foundation of China (NNSFC-61274081, $51372205,51502244)$, the China Postdoctoral Science Foundation (2014M550509), the fund of the State Key Laboratory of Solidification Processing in NWPU(SKLSP201410), and the Fundamental Research Funds for the Central Universities (3102015BJ(II)ZS014).

\section{References}

[1] Bzymek A., Application of Selected Method of Anomaly Detection in Signals Acquired During Welding Process Monitoring, Int. J. Mat. Prod. Technol., 2017, 54(4), 249.

[2] Gong X. Y., Xiao S. U.,Huang S. J. et al., Noise Characteristics of Infrared Detector Signal Acquisition System, J. Infr. Mill. Waves, 2015, 34(1), 80-86.

[3] Xie F., Liu J.,Li R. et al., A simultaneous Multiple BeiDou Signal Acquisition Algorithm for a Software-based GNSS Receiver, Optik - Int. J. Light Electron Opt., 2016, 127(4), 1607-1614.

[4] Weiss J.T., Shanks K.S.,Philipp H.T. et al., High Dynamic Range X-Ray Detector Pixel Architectures Utilizing Charge Removal, IEEE Trans. Nucl. Sci., 2016, 64(4), 1101-1107.

[5] Wojenski A., Pozniak K.T., Kasprowicz G. et al., FPGA-based GEM Detector Signal Acquisition for SXR Spectroscopy System, J. Instrum., 2016, 11(11), C11035-C11035.

[6] Tuoriniemi J., Cornelis G., Hassellöv M., New Peak Recognition Algorithm for Detection of Ultra Small Nano Particles with Single Particle ICP-MS Using Rapid Time Resolved Data Acquisition on a Sector-Field Mass Spectrometer, J. Anal. Atom. Spectr., 2015, 30(8), 1723-1729.

[7] Cheong J.W., Wu J., Dempster A., Dichotomous Search of Coarse Time Error in Collective Detection for GPS Signal Acquisition, Gps Solutions, 2015, 19(1), 61-72.

[8] Schnell M., Development of an FPGA-based Data Reduction System for the Belle II DEPFET Pixel Detector, J. Nucl. Sci. Techn., 2015, 17(4), 291-300.

[9] Viedma O., Moity N., Moreno J.M., Changes in landscape Firehazard During the Second Half of the 20th Century: Agriculture Abandonment and the Changing Role of Driving Factors, Agricult. Ecosyst. Envir., 2015, 207, 126-140.

[10] Hack E., Valzania L., Gã-Umann G. et al., Comparison of Thermal Detector Arrays for Off-Axis THz Holography and Real-Time THz Imaging, Sensors, 2016, 16(2), 221.

[11] Warren S.C., Margineanu A., Katan M. et al., Homo-FRET Based Biosensors and Their Application to Multiplexed Imaging of Signalling Events in Live Cells, Int. J. Mol. Sci., 2015, 16(7), 1469514716.

[12] Liu L., Chen C.L.P., Zhou Y. et al., A New Weighted Mean Filter with a Two-phase Detector for Removing Impulse Noise, Inf. Sci., 2015, 315(C), 1-16.

[13] Hubmayr J., Beall J., Becker D. et al., Photon-noise Limited Sensitivity in Titanium Nitride Kinetic Inductance Detectors, Appl. Phys. Lett., 2015, 106(7), 817.

[14] Powell J., Trifiro D., Cuoco E. et al., Classification methods for noise transients in advanced gravitational-wave detectors, Class. Quant. Grav., 2015, 32(21), 215012.
[15] Steven T., Siewerdsen J.H.,.Stayman J.W., Model-based Iterative Reconstruction for Flat-Panel Cone-Beam CT with Focal Spot Blur, Detector Blur, and Correlated Noise. Phys. Med. Biol., 2016, 61(1), 296-319.

[16] Wang F.P., Shui P.L., Noise-robust Color Edge Detector Using Gradient Matrix and Anisotropic Gaussian Directional Derivative Matrix, Patt. Recog., 2016, 52, 346-357.

[17] Recur B., Lewis D., Darracq F. et al., Low-frequency Noise Effect on Terahertz Tomography Using Thermal Detectors, Appl. Opt., 2015, 54(22), 6758-62.

[18] Socoró J.C., Alías F., Alsina-Pagès R.M., An Anomalous Noise Events Detector for Dynamic Road Traffic Noise Mapping in RealLife Urban and Suburban Environments, Sensors, 2017, 17(10), 2323.

[19] Arnaboldi C., Baù A.,Carniti, P., et al. Very Low Noise AC/DC Power Supply Systems for Large Detector Arrays, Rev. Sci. Instr., 2015, 86(12), 775.

[20] Xu G., Liu J.,Sun D. et al., Research on X-ray Digital Inspection Technology of Zirconium Alloy Weld, Automat. Instrum., 2015, 54(22), 6758-62.

[21] Fang M., Dai F.Z., Liu H.W. et al., Detection of Moving Targets for Wideband Radar based on Joint-sparse Recovery, J. Electr. Inform. Technol., 2015, 106(7), 817

[22] Wang L., Tang P., Yan W. et al., Influence on Power Quality and Electromagnetic Environment to Electric Vehicle Charging Pile, J. Pow. Supp., 2017, 15(C), 1-16.

[23] Ljungblad J., Hök B., Allalou A. et al., Passive In-vehicle Driver Breath Alcohol Detection Using Advanced Sensor Signal Acquisition and Fusion, Traff. Injury Prev., 2017, 18(sup1), S31.

[24] Shen Y., Wang Y., Yu X. et al., Whole-Region Hybrid Search Algorithm for DSSS Signal Acquisition, Wireless Pers. Comm., 2016, 95(2), 1-20.

[25] Zhang C., Zeng D., Jin L.I. et al., Improved algorithm for rapid detection in GNSS signal acquisition, J. Harbin Eng. Univ., 2017, 38(10), 1609-1615.

[26] Rosa R.D.L., Bruzón M.D.L.S., On the Classical and Nonclassical Symmetries of a Generalized Gardner Equation, Appl. Math. Nonlin. Sci., 2016, 1(1), 262-272.

[27] Liu Z.P., Shi Y., Zhao Y.T., Han Y.P., Niu X.T., Spatial-Temporal Evolution Characteristics and Trends of Groundwater Level in the People's Victory Canal Irrigation District, China, Appl. Ecol. Envir. Res., 2017, 15(3), 429-441.

[28] Gao W., Wang Y., Basavanagoud B., Jamil M.K., Characteristics Studies of Molecular Structures in Drugs, Saudi Pharm. J., 2017, 25(4), 580-586.

[29] Liu Z., Zhang D., Peng W., A Novel Anfis-Pso Network for Forecasting Oil Flocculated Asphaltene Weight Percentage at Wide Range of Operation Conditions, Petrol. Sci. Techn., 2018, 36(14), 1044-1050.

[30] Carreño A., Ferràndiz A.V., Ginestar D., Verdú G., Multilevel Method to Compute the Lambda Modes of the Neutron Diffusion Equation, Appl. Math. Nonlin. Sci., 2017, 2(1), 225-236.

[31] Sarma S.S.S., Garcia-Garcia G., Nandini S., Saucedo-Campos A.D., Effects of Anti-Diabetic Pharmaceuticals to Non-Target Species in Freshwater Ecosystems: A Review, J. Envir. Biol., 2017, 38(6SI), 1249-1254. 